\title{
Papers
}

\section{Diagnosis of intestinal and disseminated microsporidial infections in patients with HIV by a new rapid fluorescence technique}

T van Gool, F Snijders, P Reiss, J K M Eeftinck Schattenkerk, M A van den Bergh Weerman, J F W M Bartelsman, J J M Bruins, E U Canning, J Dankert

\begin{abstract}
Aims-To assess the value of a new rapid fluorescence method for the diagnosis of microsporidiosis in HIV seropositive patients.

Methods-Microsporidian spores in stools were demonstrated by using the fluorochrome stain Uvitex 2B. The new technique was evaluated in three groups of HIV seropositive patients with diarrhoea. Group 1: 19 patients with biopsy confirmed $E$ bieneusi infection (186 stool samples); group 2: 143 consecutive patients from whom faeces were submitted for routine investigation of diarrhoea (318 samples); group 3: 16 patients with small intestinal biopsy specimens negative for microsporidia (55 samples). The new method was used to monitor spore shedding during experimental treatment with paromomycin and albendazole in four patients.
\end{abstract}

Results-Brightly fluorescent spores were detected in all stool samples of patients in group 1. In group 216 (11\%) patients had spores in their stool samples. $E$ bieneusi was found in 11 patients; in the other five another genus of microsporidia, Encephalitozoon, was recognised. Encephalitozoon spores were also found in the urine of three of these patients and in the maxillary sinus aspirate of two of them, suggesting disseminated infection. The results were confirmed by electron microscopic examination. In group 3 negative biopsy specimens were confirmed by negative stool samples in all cases. Treatment with albendazole and paromomycin did not affect the spore shedding in three patients with $E$ bieneusi infection. By contrast, in a patient with Encephalitozoon sp infection albendazole treatment resulted in clinical improvement together with complete cessation of spore excretion in the stool.

Conclusion-The Uvitex 2B fluorescence method combines speed, sensitivity, and specificity for the diagnosis and treatment evaluation of intestinal and disseminated microsporidiosis.

(F Clin Pathol 1993;46:694-699)

Microsporidia are obligate intracellular protozoan parasites that infect most animal phyla. ${ }^{1}$ In HIV seropositive people infections with different types of microsporidia are being diagnosed increasingly often. The dominant species infecting the enterocytes of the small intestine is Enterocytozoon bieneusi, thought to be responsible for as many as $30 \%$ of cases of chronic diarrhoea for which no other microbial cause can be found. ${ }^{2-4}$ More recently, $E$ bieneusi has also been detected in the bile and biliary epithelium from patients with a clinical syndrome of AIDS related sclerosing cholangitis $^{5}$ (Abstracts presented at the 3rd European Conference on Clinical Aspects and Treatment of HIV Infection, Paris, 1992; 187, 189). Occasional infection with another microsporidian genus, Encephalitozoon, has been associated with hepatitis, ${ }^{6}$ peritonitis, ${ }^{7}$ and keratoconjunctivitis. ${ }^{8}$ Recently, a species with some of the characteristics of Encephalitozoon has been identified in the intestine of a patient with AIDS. ${ }^{9}$ Traditionally, diagnosis of $E$ bieneusi intestinal microsporidiosis has depended on invasive endoscopic procedures to provide biopsy specimens from the distal duodenum or the jejunum, for examination by light microscopy or electron microscopy. Recently, the detection of $E$ bieneusi spores in faeces has been described: this could replace endoscopic biopsy as the favoured procedure for diagnosing this infection. ${ }^{10-12}$

We initially reported a Giemsa staining procedure for faecal smears. ${ }^{10}$ Although useful, this procedure is time-consuming because it requires lengthy microscopic examination to recognise the very small spores, especially if present in low numbers. Most recently, a chromotrope-based staining technique for the detection of $E$ bieneusi in faeces has improved the discrimination between spores and other faecal elements, but the method remains laborious and lengthy to perform and has a limited sensitivity. ${ }^{12}$

To allow diagnosis of microsporidiosis to be part of routine faecal examination, we developed a new, fast, and more generally applicable fluorescence method based on staining with the fluorochrome Uvitex 2B. Uvitex $2 \mathrm{~B}$ binds to chitin, a component of the microsporidian spore wall. During intracellular maturation of spores, the chitin component of the spore wall increases. ${ }^{1}$

The method was evaluated in HIV seropositive patients with known biopsy confirmed $E$ bieneusi infection and in unselected HIV positive patients from whom faeces were routinely submitted for parasitological examination.

We were also able to use the method for 
diagnosis of intestinal and disseminated Encephalitozoon-type infections. Finally, an attempt was made to assess whether the method could be used to monitor microsporidian spore shedding during drug treatment.

\section{Methods}

The Uvitex 2B method was evaluated in three groups of HIV seropositive patients with diarrhoea.

\section{GROUP 1}

This group comprised 19 patients with biopsy confirmed $E$ bieneusi infection. In 14 the infection had been diagnosed by light microscopic examination of Giemsa stained duodenal biopsy smears. In the remaining five diagnosis was established by examining Giemsa stained faecal smears, with subsequent confirmation by biopsy. In total 186 faecal samples were collected (two to 80 samples per patient) and examined with the Uvitex 2B method. Faecal samples, which had been obtained from one patient on 40 consecutive days, were examined to study daily variation in spore shedding. All 19 patients had persistent diarrhoea and repeated tests for Salmonella sp, Shigella sp, Yersinia sp, and Campylobacter sp, as well as for parasitic pathogens, including Giardia, Cryptosporidium and Isospora spp were negative. Faecal samples were not routinely examined for mycobacteria. In 14 of 19 patients the duodenal biopsy specimens were examined for the presence of mycobacteria by Ziehl-Neelsen stain and culture; all specimens were negative.

\section{GROUP 2}

Faecal samples from $143 \mathrm{HIV}$ seropositive patients with diarrhoea, consecutively submitted for routine parasitological examination between April 1991 and February 1992, were examined with Uvitex 2B. If microsporidian spores were detected with both Uvitex 2B and Giemsa, patients were asked to undergo duodenoscopy with biopsy for confirmation of infection. In cases where a biopsy specimen was not available, electron microscopic examination of faeces was performed. Urine specimens, sinus aspirate, nasal discharge and sputum from four patients were examined. All patients had their stools routinely cultured for pathogens. In five of the 10 patients biopsied, the biopsy specimen was also examined for the presence of mycobacteria.

\section{GROUP 3}

Sixteen of the 143 patients from group 2, in whom stool examination for microsporidian spores and other pathogens was negative, were examined more extensively. Small bowel biopsies were performed either shortly after (eight patients), or before (eight patients) faecal examination with Uvitex $2 B$. These specimens were examined "blind" by light microscopy for the presence of microsporidia. The results from this group were used to assess the specificity of the new method.

\section{EVALUATION OF DRUG TREATMENT}

Parasitological and clinical response to treatment with paromomycin and albendazole were evaluated in two patients with AIDS for each drug. The patients experienced persistent diarrhoea; biopsy confirmed microsporidiosis was the only recognised microbial cause. Faecal samples were obtained daily and examined with the Uvitex $2 B$ method.

PREPARATION OF SPECIMENS FROM PATIENTS Fresh faeces $(0.5-1 \mathrm{~g})$ were homogenised in 8 $\mathrm{ml}$ distilled water and filtered through a 300 $\mu \mathrm{m}$ pore mesh sieve. After diluting the filtrate in distilled water to an appropriate density (MacFarland No 9) smears were prepared either by spinning an aliquot of $100 \mu \mathrm{l}$ in a cytospin centrifuge at $1500 \mathrm{rpm}$ for $2 \mathrm{~min}$ utes, or by air-drying $50 \mu \mathrm{l}$ on a circular $(5 \mathrm{~mm})$ area on a slide. The effects of prior disinfection of the faecal homogenates, with 4-10\% formalin, ethanol $70 \%$, or heating to $56^{\circ} \mathrm{C}$ for 30 minutes on the staining properties of spores was examined. Urine samples and maxillary sinus aspirates were centrifuged at $1250 \times g$ for 10 minutes. The pellet was washed once with phosphate buffered saline (PBS). Smears were then prepared. Small intestinal biopsy specimens were obtained by upper gastrointestinal fibreoptic endoscopy. Biopsy specimens taken from the distal part of the duodenum were processed as described before. ${ }^{4}$

Material for electron microscopy (faeces, duodenal biopsy specimens, urine, maxillary sinus aspirate, nasal discharge and sputum) was fixed in Karnovsky's fixative for $10 \mathrm{~min}$ utes, post-fixed with osmium tetroxide for 10 minutes, and embedded in Epon resin. Polymerisation was at $80^{\circ} \mathrm{C}$ for 12 hours. Sections were stained with uranyl acetate and lead citrate and examined with a Philips CM 10 electron microscope.

\section{STAINING}

The slides were fixed with methanol for 2 minutes for fluorescence staining, stained with 1\% Uvitex 2B (Ciba-Geigy Basel; PH $3.9 / \mathrm{CH} 6.25$ ) in PBS (pH 7.2) for $10 \mathrm{~min}$ utes, rinsed with PBS ( $2-5$ seconds), counterstained with $0.5 \%$ Evan's blue in PBS (30 seconds), rinsed with PBS (2-5 seconds) and left to dry at room temperature. Smears were examined at $\times 1250$ magnification under a Leitz fluorescence microscope equipped with a 50 Watt mercury high pressure lamp and an excitation filter with a transmission range from 355 to $425 \mathrm{~nm}$ and a suppression filter of $460 \mathrm{~nm}$ (Ploemopak filter block D). The active substance of Uvitex $2 B$ has a $\lambda$ light chain of $355 \mathrm{~nm}$. Uvitex 2B stained faecal smears were stored at room temperature in the dark and re-examined after six and 10 months. The average number of mature spores in five fields/slide $(\times 1250)$ was estimated.

To examine the staining properties of Uvitex 2B, smears of $E$ cuniculi obtained from 
Figure 1 Enterocytozoon bieneusi in faeces stained with Uvitex $2 B \times 3000$. Mature spores (thick arrow) and immature developmental stages (presumably sporoblasts; thin arrow). Bar $=2 \mu \mathrm{m}$

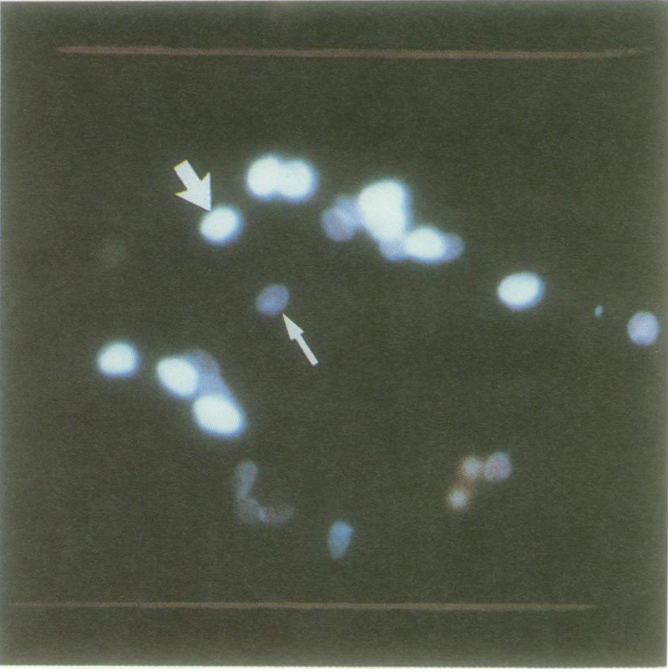

in vitro culture were prepared. $E$ cuniculi was maintained in RK13 cells with Dulbecco's minimal essential medium supplemented with $10 \%$ heat-inactivated fetal calf serum and gentamicin $(20 \mu \mathrm{g} / \mathrm{ml})$ and amphotericin B $(20 \mu \mathrm{g} / \mathrm{ml})$. The cultures were incubated at $37^{\circ} \mathrm{C}$ in an atmosphere of $5 \% \mathrm{CO}_{2}$ in air. Tissue culture supernatant fluid, containing immature stages and mature spores, was collected, washed once with PBS, pelleted, and resuspended in PBS. For smear preparation, $100 \mu \mathrm{l}$ of suspension was spun in a cytospin centrifuge at $1500 \mathrm{rpm}$ for 2 minutes.

Figure 2 Encephalitozoon sp in small intestinal enterocytes. Parasitophoras vacuoles with spores (s) in the centre, sporoblasts (sb) at the periphery and some granular material (gm) separating the stages. $B a r=1 \mu \mathrm{m}$.

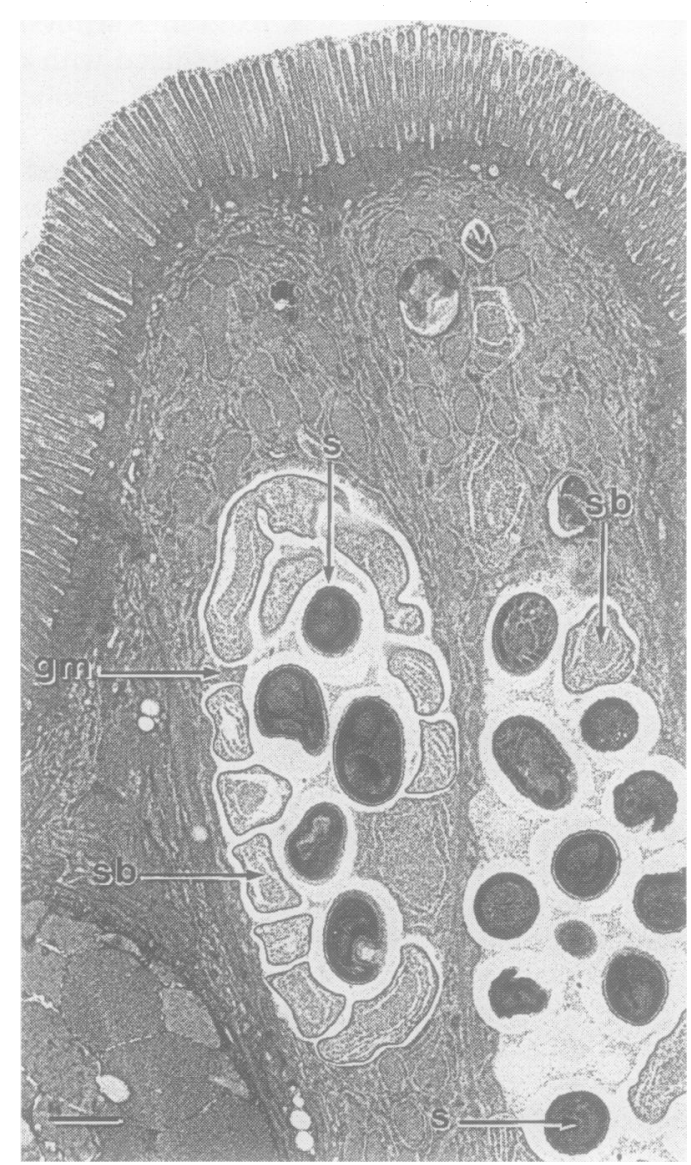

\section{Results}

STAINING PROPERTIES OF MICROSPORIDIA WITH UVITEX 2B

Uvitex 2B stained spores of $E$ cuniculi, obtained from culture supernatant fluid, fluoresced bright white. Immature developmental stages (presumably sporoblasts), appeared reddish brown. In culture supernatant fluid, mature spores considerably outnumbered immature developmental stages.

In faecal samples two different microsporidia, confirmed by electron microscopy as $E$ bieneusi and a species of Encephalitozoon, were recognised after staining with Uvitex 2B. The same staining properties-namely bright white fluorescence or reddish brownas observed with the cultured $E$ cuniculi, were exhibited respectively by mature and immature stages of both species. In the faecal smears, however, the immature stages greatly outnumbered the mature spores.

The shape and size of mature spores of $E$ bieneusi (broadly oval, about $1.6 \times 1.0 \mu \mathrm{m}$ ) showed little variation (fig 1), but the larger spores of Encephalitozoon varied considerably, both in shape and size, being broad and rodlike or kidney shaped, and measuring 2.5-3.3 $\times 1 \cdot 3-2 \cdot 1 \mu \mathrm{m}$. Mature spores and immature developmental stages of Encephalitozoon $\mathrm{sp}$ were also seen in urine samples, nasal discharge, maxillary sinus aspirate, and sputum. A morphological characteristic, always present in a proportion of the mature spores of $E$ bieneusi, and (less frequently) in those of Encephalitozoon sp, was a concave inner stripe and shadow. This characteristic was used throughout the study as an important criterion for identification of microsporidian spores. The inner stripe and shadow were not observed in mature spores of $E$ cuniculi derived from culture supernatant fluids.

The background was dark brown or black and discrimination between microsporidian spores and fluorescing structures such as yeasts and fungi was possible on the basis of clearly visible differences in size and morphology. Only small fungal spores showed some resemblance to $E$ bieneusi and Encephalitozoon sp spores, but they were more rod-like and the concave inner stripe and shadow were always absent.

The time needed to read the slides varied from 30 seconds to 2 minutes; to exclude microsporidia infection a 3 minute search was sufficient.

Fixation with ethanol $70 \%$ or heating of faecal suspensions to $56^{\circ} \mathrm{C}$ for 30 minutes did not alter the staining properties of mature spores, but fixation with formalin reduced considerably the intensity of fluorescence of mature spores.

Storage of Uvitex 2B stained smears at room temperature in the dark for six months did not influence fluorescence of mature spores but a slight decrease of intensity was observed after 10 months.

Electron microscopic examination of Encephalitozoon infections in small intestinal biopsy specimens showed all developmental stages other than sporoplasms (fig 2). 

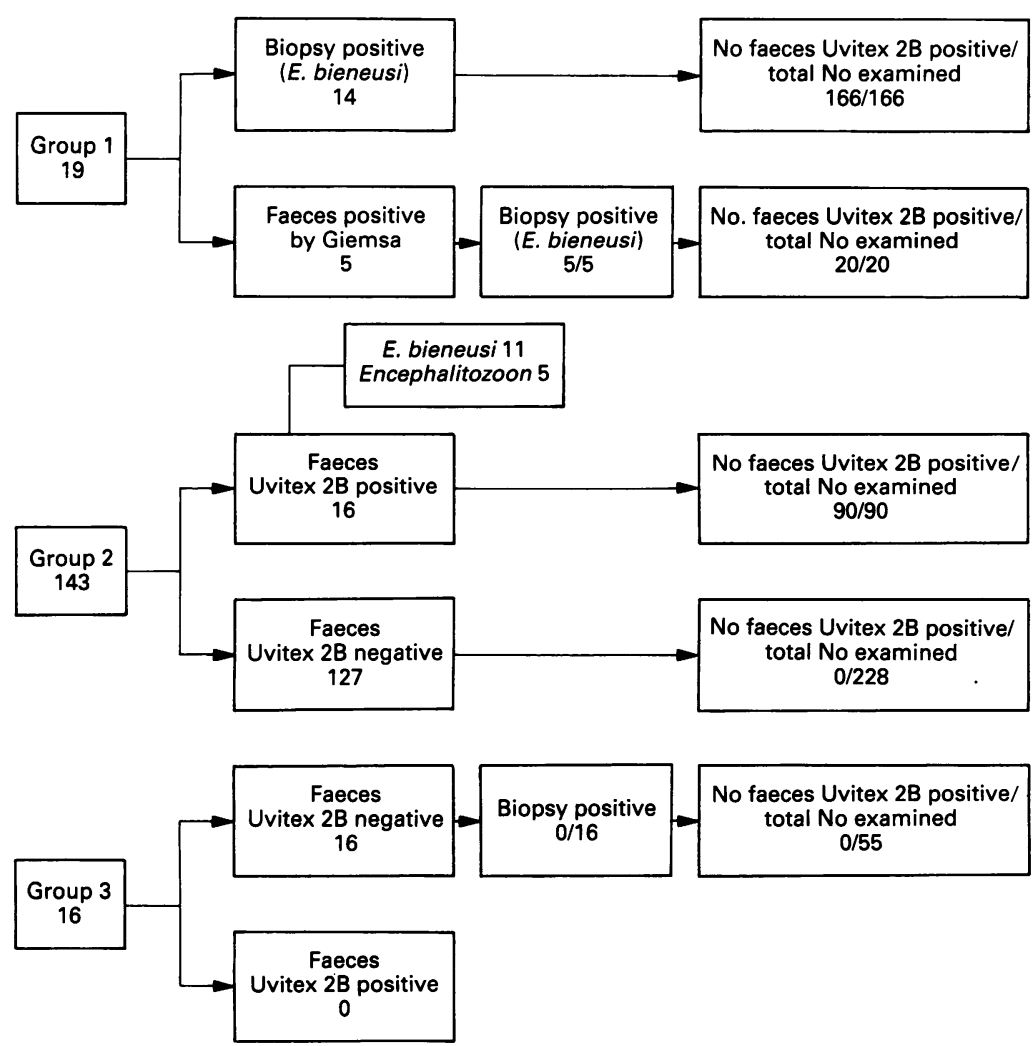

Figure 3 Results of faeces and duodenal biopsy examination in patients with biopsy confirmed $E$ bieneusi infection (group 1), with diarrhoea of unknown origin routinely submitted for parasitological examination (group 2), and with negative biopsy specimens for microsporidia (group 3).
Meronts were found lying adjacent to the membrane of a parasitophoras vacuole; sporogon phases were found lying free within the vacuole. Around the parasites in the vacuole a variable amount of loose granular material was frequently observed. Sporogony was disporous, not in a sporophorous vesicle, and the nuclear arrangement was monokaryotic. These characteristics are diagnostic of the genus Encephalitozoon.

\section{DIAGNOSIS OF MICROSPORIDIOSIS WITH UVITEX} 2B IN THE THREE GROUPS

Group 1

Mature spores and immature stages were seen in all 186 Uvitex 2B stained faecal smears from the 19 patients with known $E$ bieneusi small intestinal infection (fig 3). Faecal samples obtained from one patient on 40 consecutive days were all positive. A varia-

Examination of various specimens from five patients with AIDS by Uvitex $2 B$, electron microscopy or light microscopy for Encephalitozoon sp, infection

\begin{tabular}{|c|c|c|c|c|c|}
\hline \multirow[b]{3}{*}{$\begin{array}{l}\text { Case } \\
\text { No }\end{array}$} & \multicolumn{5}{|c|}{ Specimen examined: } \\
\hline & \multicolumn{2}{|l|}{ Faeces } & \multirow{2}{*}{$\begin{array}{l}\begin{array}{l}\text { Duodenal } \\
\text { biopsy }\end{array} \\
\text { LM and EM }\end{array}$} & \multirow{2}{*}{$\begin{array}{l}\text { Urine } \\
\text { Uvitex } 2 B \\
\text { and } E M\end{array}$} & \multirow{2}{*}{$\begin{array}{l}\text { Maxillary sinus } \\
\text { aspirate/nasal discharg } \\
\text { Uvitex } 2 B \\
\text { and } E M\end{array}$} \\
\hline & Uvitex $2 B$ & $E M$ & & & \\
\hline $\begin{array}{l}1 \\
2 \\
3 \\
4 \\
5 \dagger\end{array}$ & $\begin{array}{l}+ \\
+ \\
+ \\
+ \\
+\end{array}$ & $\begin{array}{l}+ \\
\text { ND } \\
+ \\
+ \\
\text { ND }\end{array}$ & $\begin{array}{l}+ \\
+ \\
+ \\
+ \\
\text { ND }\end{array}$ & $\begin{array}{l}+ \\
+\star \\
+ \\
+ \\
\text { ND }\end{array}$ & $\begin{array}{l}\text { ND } \\
+ \\
\text { ND } \\
+ \\
+ \\
\text { ND }\end{array}$ \\
\hline
\end{tabular}

tion in spore numbers of 1-30 mature spores/field $(\times 1250)$ was observed; no regular daily or weekly pattern was discernible.

\section{Group 2}

Intestinal infection-Using Uvitex $2 \mathrm{~B}$, intestinal microsporidiosis was diagnosed in 16 of $143(11 \cdot 2 \%)$ patients with diarrhoea. $E$ bieneusi was found in 11 of $16(69 \%)$, and Encephalitozoon infection in five of $16(31 \%)$ patients (fig 3). Concomitant (potential) pathogenic bacteria and parasites were detected in the stools in six of 16 patients; Entamoeba histolytica (twice), Entamoeba histolytica, and Campylobacter jejuni (once), Blastocystis hominis (once), Shigella flexneri (once) and Mycobacterium avium intracellulare (once). All duodenal biopsy specimens examined for mycobacteria were negative.

In patients with $E$ bieneusi infection confirmation was obtained by light microscopic examination of duodenal biopsy specimens in six cases and by electron microscopic examination of faecal samples from the remaining five cases. In one patient with low numbers of $E$ bieneusi spores in faeces, confirmed by electron microscopy, microsporidia were not detected in biopsy specimens either by light microscopic or electron microscopy. In two other patients, with numerous spores in the stool, extensive light microscopic examination of duodenal biopsy smears was required before any infected cell was detected.

Biopsy specimens for electron microscopy examination were available from four of the five patients in whom Encephalitozoon had been found in the faeces with Uvitex 2B; Encephalitozoon infection was confirmed in three (table). In the fourth patient no microsporidia were detected in biopsy specimens even after prolonged examination by both light microscopic and electron microscopy (case 4). The CD4 + counts in the five patients with Encephalitozoon sp infection ranged between 0.01 and $0.14 \times 10^{9} / 1$.

Extraintestinal infection-Urine samples were examined from four of the five patients in whom Encephalitozoon sp had been found as an intestinal infection. In three of them spores and immature stages characteristics of Encephalitozoon sp were detected with Uvitex 2B; electron microscopic confirmation was obtained in two (table). Parasites were located both in extracellular as well as in intracellular positions. The morphology of infected epithelial cells by light microscopy suggested that they had originated from the lower urinary tract. There was no clinical or laboratory evidence for the existence of urinary tract disease. Bacterial cultures from urine were negative.

In two patients with an Encephalitozoon infection (cases 2 and 4) spores were also detected in maxillary sinus aspirate, nasal discharge, and sputum. Ultrastructurally, the mature spores and immature developmental stages observed seemed to be identical with those present in duodenal biopsy specimens. 
Group 3

In this group of 16 patients with diarrhoea light microscopy of small intestinal biopsy specimens did not provide evidence of microsporidiosis in any of the patients. A total of 55 stool samples (two to 10 per patient) were examined with negative results (fig 3).

\section{EVALUATION OF DRUG TREATMENT}

Two patients with intestinal $E$ bieneusi infection as the sole known cause of their chronic diarrhoea were treated with oral paromomycin $500 \mathrm{mg}$ three times a day for 12 days. Both patients experienced a decrease in diarrhoeal symptoms, but spores were detected in daily stool samples throughout the treatment period without obvious alteration in the shedding pattern of the spores.

One patient with $E$ bieneusi-associated chronic diarrhoea was treated with oral albendazole $400 \mathrm{mg}$ twice a day for 15 days. This did not result in any changes, either in diarrhoeal signs and symptoms, or in shedding of spores in faeces. Another patient with chronic diarrhoea and biopsy confirmed Encephalitozoon infection of the small intestine with massive spore excretion in the stool received oral albendazole $400 \mathrm{mg}$ twice a day. His diarrhoea lessened in a few days and fully disappeared after three weeks. Spore excretion stopped completely after six days of treatment and the stool remained negative for two months. A duodenal biopsy specimen taken after two months treatment was free of parasites. After 10 weeks, in spite of continued albendazole treatment, diarrhoea recurred and stool examination at 16 weeks contained Encephalitozoon spores again, confirming the clinical relapse.

\section{Discussion}

The diagnosis of $E$ bieneusi intestinal microsporidiosis has, until recently, depended on invasive endoscopic procedures. ${ }^{2-413}$ Noninvasive means for diagnosis became feasible after spores had been recognised in faeces. ${ }^{12-14}$ Unfortunately, Giemsa stained faecal preparations require experienced interpretation. Although a recently described chromotrope based staining technique ${ }^{14}$ may overcome these restrictions to some extent, the lengthy ( 2 hours) and laborious staining procedure makes it unsuitable for routine use. The Uvitex $2 \mathrm{~B}$ method is a considerable advance on previous methods for the diagnosis of microsporidial infections because of its simplicity and the brilliant white fluorescence which facilitates rapid recognition of spores. The staining procedure takes less than 15 minutes to perform, and the time to read the slides reliably is only of the order of minutes. The staining properties are not reduced by the use of ethanol $70 \%$ or heating to $56^{\circ} \mathrm{C}$ for 30 minutes. Therefore, these methods for disinfection of the specimens can be used before they are processed further in the laboratory.

As Uvitex 2B binds to chitin, it is not strictly specific for microsporidia. ${ }^{14}$ The microsporidian spores, however, can easily be differentiated by morphological characteristics from other chitin-containing microorganisms.

A valuable feature of the Uvitex 2B method is that it distinguishes between mature chitin-containing spores and noninfective immature spores of both $E$ bieneus $i$ and Encephalitozoon sp. Mature spores of $E$ cuniculi were abundantly present in supernatant fluid from in vitro cultures. In contrast, immature spores of $E$ bieneusi and Encephalitozoon greatly outnumbered mature spores in faeces. This suggests that the lifespan of small intestinal epithelial cells in patients with microsporidiosis is too short for complete development of most of the microsporidia. The concave inner stripe and shadow observed in a large part of the mature spores probably represents a partly collapsed inner spore wall resulting from discharge of the spore contents into the environment.

In all patients with intestinal microsporidiosis, spores were detected with the Uvitex 2B method in all faecal specimens, irrespective of day to day variation in spore shedding and faeces consistency. This is in contrast with observations by Weber et al, who, using the chromotrope-based staining method in two patients with intestinal microsporidiosis, found only five of 25 faecal samples to be positive. ${ }^{12}$

Furthermore, some studies suggest a higher sensitivity of Uvitex $2 \mathrm{~B}$ compared with small intestinal biopsy for diagnosis of intestinal microsporidiosis. In two patients stool examination with the Uvitex 2B method revealed microsporidia (confirmed as $E$ bieneusi and Encephalitozoon sp by electron microscopy of stool and urine sediment, respectively), while duodenal biopsy specimens obtained subsequently were negative by both light microscopy and electron microscopy. In two other patients with numerous spores of $E$ bieneusi in their stools parasites could be found in biopsy specimens only after a prolonged search at light microscopy.

In previous studies with HIV seropositive patients with chronic diarrhoea not explained by other intestinal pathogens, microsporidiosis has ranged from 20 to $30 \% .^{2-4}$ The prevalence of $11 \%$ ( $E$ bieneusi and Encephalitozoon sp combined) found in our study reflects the prevalence of intestinal microsporidiosis in unselected HIV positive patients presenting with diarrhoea.

Until now small intestinal infection with $E$ bieneusi has been the predominant type of microsporidiosis in HIV seropositive individuals. Our staining technique also led to the recognition of small intestinal infection with another genus of microsporidia, which could be distinguished from $E$ bieneusi on the basis of differences in size and shape, and which was ultrastructurally similar to Encephalitozoon.

Serological surveys suggest that antibodies to $E$ cuniculi are relatively common among certain human populations, with rates up to 
$20 \%$ in HIV seropositive individuals. ${ }^{15}$ The identification of Encephalitozoon sp in five of the $16(31 \%)$ patients with intestinal microsporidiosis indicates the potential importance of this genus as a cause of chronic diarrhoea in HIV positive subjects. As has been reported for $E$ bieneusi in this study and others, ${ }^{412}$ infections with Encephalitozoon sp were found only in patients with greatly reduced CD4 + lymphocyte numbers.

Whether the tendency of the Encephalitozoon $\mathrm{sp}$ to disseminate beyond the gut will result in increased recognition of new clinical syndromes remains to be elucidated. A recent study suggests that Encephalitozoon-like micro-organisms can cause renal pathology in patients with AIDS. ${ }^{16}$ The presence of Encephalitozoon spores in sputum as observed in two patients in our study does not prove pulmonary infection by microsporidia as postnasal drip may be mixed with the sputum.

Currently, no proved effective treatment for human microsporidial infections exists. In HIV seropositive patients with $E$ bieneusi intestinal infection clinical improvement of diarrhoeal signs and symptoms has been reported in uncontrolled studies with a small number of patients treated with metronidazole $^{4}$ or albendazole. ${ }^{17}$ Neither drug eradicated the parasites from the small intestine, although ultrastructural changes in $E$ bieneusi in biopsy specimens following albendazole treatment have been reported. ${ }^{19}$ Paromomycin, an aminoglycoside which shows minimal absorption after oral administration, has recently shown promise for the treatment of cryptosporidial diarrhoea in HIV seropositive patients. ${ }^{18}$

In the patients with microsporidiosis due to $E$ bieneusi who were treated with paromomycin or albendazole, no changes in the shedding pattern of spores in stools were seen; although some clinical improvement was observed with paromomycin. This effect could be the result of changes in the intestinal flora by the drug, leading to reduction of intraluminal digestion of malabsorbed nutrients and thus stool volume. An effect of paromomycin on another unrecognised pathogen also cannot be excluded.

In contrast, in the patient with Encephalitozoon infection treatment with albendazole led to clinical improvement as well as cessation of spore shedding in the stool. Recently, we made a similar observation in another patient with Encephalitozoon infection (case 4), with complete cessation of spore shedding in faẹces and also in urine and sinus discharge. These findings suggest that albendazole may well exert a different effect on Encephalitozoon and $E$ bieneusi in vivo.

Our new fast and easy staining technique with Uvitex 2B will bring the diagnosis of microsporidial infections within the range of routine practice. Furthermore, it may facilitate research regarding epidemiology, pathogenesis, and treatment of microsporidiosis.

We thank Ms JM de Bruin and Mr H Gilis for technical assistance, Dr JCM Vetter and Dr J Veenstra for advice and clinical support, and Dr LM Kortbeek (National Institute of Public Health and Environmental Protection, Bilthoven) for providing E cuniculi for culture.

1 Canning EU, Lom J. The microsporidia of vertebrates. New York: Academic Press, 1986.

2 Peacock CS, Blanshard C, Tovey DG, Ellis DS, Gazzard BG. Histological diagnosis of intestinal microsporidiosis in patients with AIDS. F Clin Pathol 1991;44:558-63.

3 Orenstein JM, Chiang J, Steinberg W, Smith PD, Rotterdam H, Kotler DP. Intestinal microsporidiosis as Rotterdam H, Kotler DP. Intestinal microsporidiosis as a cause of diarrhoea in human immunodeficiency virus1990;21:475-81.

4 Eeftinck Schattenkerk JKM, van Gool T, van Ketel RJ, et al. Clinical significance of small-intestinal microsporidiosis in HIV-1-infected individuals. Lancet 1991, 337:895-8.

5 McWhinney PHM, Nathwani D, Green JF, et al. Microsporidiosis detected in association with AIDSrelated sclerosing cholangitis. AIDS 1991;5:1394-5.

6 Terada S, Reddy RK, Jeffers LJ, Cali A, Schiff ER Microsporidian hepatitis in the acquired immunodeficiency syndrome. Ann Intern Med 1987;107:61-2

7 Zender HO, Arrigoni E, Eckert J, Kapanci Y. A case of Encephalitozoon cuniculi peritonitis in a patient with Encephalitozoon cuniculi peritonitis in
AIDS. Am $\mathcal{F}$ Clin Pathol 1989;92:352-6.

8 Didier ES, Didier PJ, Friedberg DN, et al. Isolation of a new human microsporidian, Encephalitozoon hellem (n.sp.) from three AIDS patients with keratoconjunctivitis. F Infect Dis 1991;163:617-21.

9 Cali A, Orenstein JM, Kotler DP, Owen R. A comparison of two microsporidian parasites in enterocytes of AIDS patients with chronic diarrhoea 7 Protozool 1991;38: 96-8.

10 Van Gool T, Hollister WS, Eeftinck Schattenkerk J, et al. Diagnosis of Enterocytozoon bieneusi microsporidiosis in Diagnosis of Enterocytozoon bieneusi microsporidiosis in 1990;336:697-8.

11 Orenstein JM, Zierdt W, Zierdt C, Kotler DP. Identification of spores of Enterocytozoon in stool and duodenal fluid from AIDS patients. Lancet 1990;336: 1127-8.

12 Weber R, Bryan RT, Owen RL, et al. Improved lightmicroscopical detection of microsporidia spores in stool and duodenal aspirates. $N$ Engl F Med 1992;326:161-6.

13 Desportes I, le Charpentier Y, Galian A, et al. Occurrence of a new microsporidian: Enterocytozoon bieneusi n.g. n. sp., in the enterocytes of a human patient with AIDS. n. sp., in the enterocytes of

14 Koch HH, Pimsler M. Evaluation of Uvitex 2B: a nonspecific fluorescent stain for the detection and identifying of fungi and algae in tissue. Lab Med 1987;18:603-6.

15 Hollister WS, Canning EU, Willcox A. Evidence for widespread occurrence of antibodies to Encephalitozoon cuniculi (microspora) in man provided by ELISA an other serological tests. Parasitology 1989;102:33-43.

16 Orenstein JM, Dieterich T, Kotler DP. Systemic dissemination by a newly recognized intestinal microsporidi species in AIDS. AIDS 1992;6:1143-50.

17 Blanshard C, Ellis DS, Tovey DG, et al. Treatment of intestinal microsporidiosis with albendazole in patients with AIDS. AIDS 1992;6:311-3.

18 Clezy K, Gold J, Blaze J, Jones P. Paromomycin for the treatment of cryptosporidial diarrhoea in AIDS patients. AIDS 1991;5:9. 